\title{
Development of a Dual-Label Time-Resolved Fluorescence Immunoassay (TRFIA) for Screening of Bladder Cancer based on Simultaneous Detection of BLCA-4 and NMP52 in Urine
}

\author{
Xiaozhu Liu ${ }^{1}$, Yinfeng Li ${ }^{1}$, Yan Wang ${ }^{2}$, Wenqiao Sun ${ }^{3}$, Laiqing $\mathrm{Li}^{4}$ and Licheng Zhang ${ }^{3^{*}}$ \\ ${ }^{1}$ Guizhou Institute of Technology, Guiyang, 550000, China \\ ${ }^{2}$ Shangdong Medicine Technician College, Tai'an, 271000, Shandong, China \\ ${ }^{3}$ Cancer Center of the 88 Hospital of People's Liberation Army, Tai'an, 271000, Shandong. China \\ ${ }^{4}$ Guangzhou Youdi Biotechnology Company, Guangzhou, 510000, Guangdong, China
}

\begin{abstract}
Bladder cancer is a heterogeneous disease and occupies highest incidence in developed country. Time-resolved fluoroimmunoassays (TRFIA) is a new detection technique with a feature of sensitive, simple and inexpensive. The aim of this study is to establish a dual-label TRFIA for the simultaneous detection of BLCA-4 and NMP52 in urine in a single run. The sandwich immunoassay was used to detect the concentration of BLCA-4 and NMP52 in urine. BLCA4 and NMP52 in urine were captured by anti-BLCA-4 and anti-NMP52 antibodies immobilized on microtiter wells, and then banded together with another anti-BLCA-4 and anti-NMP52 labeled with europium(III) $\mathrm{Sm}^{3+}$ and samarium(III) $\mathrm{Eu}^{3+}$ chelates, followed by fluorescence measurement using time resolved fluorometry. To assess the performance of this assay, clinical urine samples were used, and then commercialized kits were compared. The sensitivity for BLCA-4 and NMP52 detection of this assay were $2 \mathrm{U} / \mathrm{mL}$ (dynamic range, 5-300 U/L) and $1 \mu \mathrm{g} / \mathrm{ml}$ (dynamic range, $2-150 \mu \mathrm{g} / \mathrm{ml}$ ) respectively. The correlation coefficients $(R)$ between the present dual-label TRFIA and commercially available kits were high. R-values were 0.99 for BLCA-4 and NMP52. The cross-reactivity seems not to influence the results. The present dual-label TRFIA, allowing the simultaneous detection ofBLCA-4 and NMP52, has high sensitivity, specificity, and accuracy in clinical sample analysis. Therefore, it has good prospects of application.
\end{abstract}

Keywords: Bladder cancer; BLCA-4; Dual-label TRFIA; NMP52

\section{Introduction}

Bladder cancer is a heterogeneous disease and occupies highest incidence in developed country [1,2]. There are over 70,000 new cases of bladder cancer each year in the United States alone [3]. The incidence of bladder cancer rises with age, peakingbetween age 50 years and 70 years, and is three times more common in men than in women [4]. The high rate of recurrence is the feature of bladder cancer that makes effective detection means is crucial importance for bladder cancer patients.

The conventional method for bladder cancer detection is morphological examination of cytology samples or cystoscopic biopsies [5]. Urine cytology is poorly differentiated tumors and time-consuming practice for both the patient and physician [6,7]. The diagnosis and monitoring of bladder cancer are facilitated by the availability of newer, simpler urine based diagnostic tests $[2,8,9]$. Recently, a number of molecular markers, such as BLCA-4, NMP52 and chromosomal aberrations, have been studied extensively by various groups [1013]. BLCA- 4 isone of six nuclear matrix proteins that are specifically expressed in bladder cancer [14]. Overexpression of BLCA- 4 causes cells to express a more tumorigenic phenotype [15]. ELISA analysis showed that sensitivity and specificity of BLCA-4 for bladder cancer detection was $96.4 \%$ and $100 \%$ respectively $[11,12,16]$. High-level of sensitivity and specificity makes BLCA- 4 to be a better candidate for detecting bladder cancer. It has been demonstrated that the concentration of NMP52, one of nuclear matrix proteins, was greater in bladder cancer cell lines than in the normal bladder [17]. Evidences proved that sensitivity of NMP52 for bladder cancer test was $94 \%$ and specificity was $95 \%[10,18]$. Therefore, NMP52 could be used as a diagnostic tool for screening bladder cancer owing to its high sensitivity and specificity.

At present, many commercialized kits are available to detect urinary levels of BLCA-4 and NMP52. But they need two steps to detect these two markers. In addition, they have some other limitations, such as, time and labor consuming as well as high cost. Time-resolved fluoroimmunoassays (TRFIA) is a new detection technique with a feature of sensitive, simple and inexpensive [19]. TRFIA employs lanthanide chelates as fluorescent labels which offer the advantages of long fluorescence lifetimes and high quantum yields [20]. In a timeresolved manner the specific signal is detected after the disappearance of short-lived, nonspecific background fluorescence. The aim of this study is to establish a dual-label TRFIA for the simultaneous detection of BLCA-4 and NMP52 in urine in a single run (Figure 1).

\section{Materials and Methods}

\section{Materials}

The BLCA-4 and NMP52 standards were supplied by Abcam (Cambridge, MA). The monoclonal antibody (McAbs) against BLCA4 and NMP52 were got from Hotgen Biotechnical Corporation. BSA was obtained from Merck (Darmstadt, Germany). $\mathrm{Sm}^{3+}$ - and $\mathrm{Eu}^{3+}$ labeling kits were purchased from PerkinElmer (Norwalk, CT, USA). Enzyme-linked immuno sorbent assay kits for BLCA-4 and NMP52

*Corresponding author: Licheng Zhang, PhD, Cancer Center of the 88 Hospital of People's Liberation Army, Humen Road 6, Tai'an, 271000, Shandong, China, Tel: +8602022056980; Fax: +8602022056980; E-mail: zhang88cn@sohu.com

Received March 27, 2017; Accepted April 10, 2017; Published April 12, 2017

Citation: Liu X, Li Y, Wang Y, Sun W, Li L, et al. (2017) Development of a DualLabel Time-Resolved Fluorescence Immunoassay (TRFIA) for Screening of Bladder Cancer based on Simultaneous Detection of BLCA-4 and NMP52 in Urine. J Bioprocess Biotech 7: 303. doi:10.4172/2155-9821.1000303

Copyright: ( 2017 Liu X, et al. This is an open-access article distributed unde the terms of the Creative Commons Attribution License, which permits unrestricted use, distribution, and reproduction in any medium, provided the original author and source are credited. 

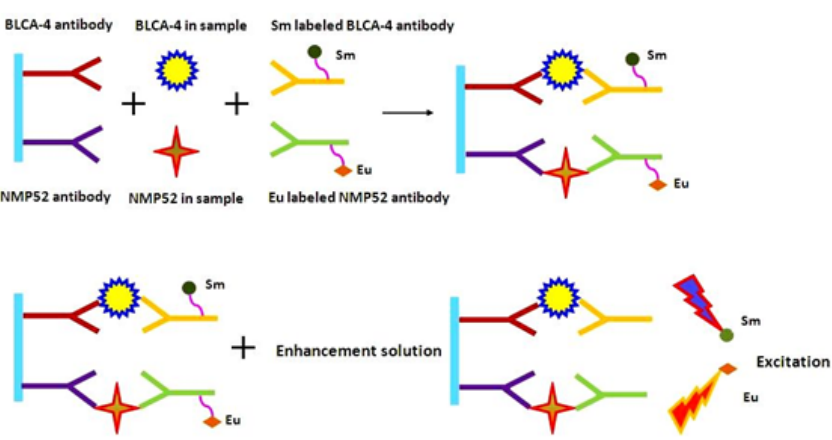

Figure 1: Schema of the dual-label TRFIA for BLCA-4 and MNP52 detection.

were purchased from Roche Diagnostics GmbH (Mannhein, Germany). Centrifugal filters with molecular weight cut off $10 \mathrm{KDa}$ and $60 \mathrm{KDa}$ were purchased from Millipore (Bedford, MA). Sephadex G50 column was obtained from GE Healthcare (Uppsala, Sweden). Tris andTriton X-100 were obtained from Sigma-Aldrich (St. Louis, MO, USA). Other reagents were of analytical grade and were supplied by Beijing Reagent Corporation (Beijing, China).

\section{Coating of the micro well plates}

Monoclonal antibody for BCLA-4 and NMP52 were diluted to final concentration of $1 \mathrm{mg} / \mathrm{L}$ with $50 \mathrm{mmol} / \mathrm{L}$ carbonate ( $\mathrm{pH} 9.6$ ) buffer. Then, $100 \mu \mathrm{L}$ of the diluted monoclonal antibody for BCLA- 4 and NMP52 was added to each well for double coating followed by incubation at $4^{\circ} \mathrm{C}$ overnight. After coating, the coating buffer was discarded, the plates were washed 3 times with PBS containing 0.05\% Tween-20 (v/v) then blocked with blocking buffer $(50 \mathrm{mmol} / \mathrm{L}$ Tris- $\mathrm{HCl}$ containing $3 \% \mathrm{BSA}$, $\mathrm{m} / \mathrm{v}, \mathrm{pH} 8.0$ ) overnight at $4^{\circ} \mathrm{C}$. The blocking bufferwas discarded. The plate was vacuum dried and stored at $-20^{\circ} \mathrm{C}$ until use.

\section{Labeling antibody with $\mathrm{Sm}^{3+}$ and $\mathrm{Eu}^{3+}$ chelates}

Antibody labeling with $\mathrm{Sm}^{3+}$ and $\mathrm{Eu}^{3+}$ chelates were carried out using $\mathrm{Sm}^{3+}$ and $\mathrm{Eu}^{3+}$ labeling kits according to the manufacturer's instructions. In brief, $1 \mathrm{mg}$ of McAbs(BLCA-4) was washed 3 times using labeling buffer $\left(50 \mathrm{mmol} / \mathrm{L} \mathrm{Na}_{2} \mathrm{CO}_{3}, \mathrm{pH}\right.$ 9.0) and collected through centrifugal filters. Then, it was suspended in $250 \mu \mathrm{L}$ of labeling buffer, and mixed gently with $500 \mu \mathrm{g}$ of $\mathrm{Sm}^{3+}$ chelates in $250 \mu \mathrm{L}$ of the same buffer. The mixture was incubated overnight at room temperature, and collected using Sephadex G50 column equilibrated with $50 \mathrm{mmol} / \mathrm{L}$ Tris- $\mathrm{HCl}$ buffer ( $\mathrm{pH} 7.8$ ) containing $9 \mathrm{~g} / \mathrm{L} \mathrm{NaCl}$. Store the conjugated McAbs(BLCA-4)Tris- $\mathrm{HCl}$ buffer $(50 \mathrm{mmol} / \mathrm{L}, 0.1 \% \mathrm{BSA}, \mathrm{m} / \mathrm{v}, \mathrm{pH} 7.8$ ) at $4^{\circ} \mathrm{C}$. McAbs (NMP52) was labeled with $\mathrm{Eu}^{3+}$ by the same procedure used for $\mathrm{Eu}^{3+}$.

\section{Assay procedures}

We first added $25 \mu \mathrm{L}$ of standards or clinical urine samples into the coated wells, and then added $200 \mu \mathrm{L}$ of $\mathrm{Sm}^{3+}$ labeled BCLA- 4 and $\mathrm{Eu}^{3+}$ labeled NMP52 antibody $(1 \mu \mathrm{g} / \mathrm{mL})$. The plate was incubated at room temperature for $1 \mathrm{~h}$. After 4 times of washing, we put $200 \mu \mathrm{L}$ of enhancement solution $(15 \mu \mathrm{mol} / \mathrm{L} \beta$-NTA, $50 \mu \mathrm{mol} / \mathrm{L}$ TOPO and $0.1 \%$ Triton X-100, v/v) into each well, gently shake the plate for $5 \mathrm{~min}$ and read the fluorescence values by fluorescence readings (Auto DELFIA 1235, PerkinElmer).

\section{Evaluation of the assay}

We evaluated the sensitivity of the assay using a serial standard dilutions of BLCA-4 (0 U/mL, $5 \mathrm{U} / \mu \mathrm{g}, 10 \mathrm{U} / \mu \mathrm{g}, 25 \mathrm{U} / \mu \mathrm{g}, 50 \mathrm{U} / \mu \mathrm{g}, 100 \mathrm{U} / \mu \mathrm{g}, 200 \mathrm{U} / \mu \mathrm{g}$, $300 \mathrm{U} / \mu \mathrm{g})$, and NMP52 $(0 \mu \mathrm{g} / \mathrm{ml}, 1 \mu \mathrm{g} / \mathrm{ml}, 5 \mu \mathrm{g} / \mathrm{ml}, 10 \mu \mathrm{g} / \mathrm{ml}, 20 \mu \mathrm{g} / \mathrm{ml}, 40$ $\mu \mathrm{g} / \mathrm{ml}, 80 \mu \mathrm{g} / \mathrm{ml}, 150 \mu \mathrm{g} / \mathrm{ml}$ ). First, we assessed the averages (X) and standard deviations (SD) from twenty independent experiments $(n=20)$. Next, we analyzed the low, median and high grade of clinical samples to calculate the accuracy of the assay. Then, we evaluated the intra- and inter- assay variations from ten independent experiments $(n=10)$. Finally, we estimated the recovery using 5 urine samples at different analyte levels. The recoveries (\%) were established using the equation: Recovery $=100 \times$ (measured value/ original concentration). For the specificity tests, we evaluated different concentrations of thyroid hormones (TH), luteinizing hormone (LH), testosterone, estradiol and cortisol and follicle stimulating hormone (FSH).

\section{Comparison of TRFIA with commercialized kits}

We compared the TRFIA method with BCLA-4 and NMP52 enzymelinked immuno sorbent assay kits as parallel tests. The appropriate controls were within the ranges provided by the manufacturer for all runs. We analyzed 175 clinical urine samples, 85 from Zhujiang Hospital (Guangzhou, China) and 90 from Jinan Hospital (Jinan, China). The enzyme-linked immuno sorbent test was carried out according to the manufacturer's instructions.

We constructed the receiver operator characteristic (ROC) curves, and calculated the area under the curve (ROC- AUC) with a 95\% confidence interval using 360 samples from Zhujiang Hospital. Sensitivity and specificity were calculated independently using voided urine samples of patients with pathologically confirmed bladder cancer. Subgroups of bladder cancer were assessed from the histological evidences (subtype, stage and grade of the tumor).

\section{Preparation of urine samples}

This study approved by the ethics committee of Zhujiang Hospital (REC number: GDYY205427C) and Jinan Hospital (REC number: SDYY306236C). Urine samples from patients with pathologically confirmed bladder cancer, cystitis patients and normal controls were collected from Zhujiang Hospital and Jinan Hospital. All samples were morning voiding urines, bar coded, and stored in the $-20^{\circ} \mathrm{C}$ freezer. Infected urine samples were from patients excluded from urologic tumors.

\section{Statistical analysis}

The data were shown as mean \pm standard deviation (mean \pm $\mathrm{SD}$ ). Pearson's linear regression was used to present the linearity and correlations. Urine samples analyzed using TRFIA method and a commercial ELASA kit were compared using the paired Student'stest by SPSS 19.0 (Chicago, IL, USA), and $\mathrm{P}<0.05$ was considered as statistically significant.

\section{Results}

\section{Demographic data of patients}

A total of 360 patients (240 males and 120 females) and 40 healthy individuals were enrolled in this study.54 (15\%) patients were diagnosed as cystitis, $126(35 \%)$ patients were diagnosed as benign disease and 180 (50\%) patients were diagnosed malignant bladder cancer. The histopathological grade of bladder cancer patients were Ta stage $(\mathrm{n}=25$, $8 \%)$, T1 stage $(n=21,7 \%)$, T2 stage $(n=98,32 \%)$, T3 stage $(n=80,26 \%)$ and T4 stage $(n=82,27 \%)$ refer to the WHO classification system.

The standard curves plotted for BLCA-4 and NMP52 were obtained 
Citation: Liu X, Li Y, Wang Y, Sun W, Li L, et al. (2017) Development of a Dual-Label Time-Resolved Fluorescence Immunoassay (TRFIA) for Screening of Bladder Cancer based on Simultaneous Detection of BLCA-4 and NMP52 in Urine. J Bioprocess Biotech 7: 303. doi:10.4172/21559821.1000303

and shown in Figure 2. The working concentration ranges for BLCA4 and NMP52 were 5-300 $\mathrm{U} / \mathrm{ml}$ and $2-150 \mu \mathrm{g} / \mathrm{ml}$ respectively. The resulting calibration plots exhibited well-defined linear relationships between the analyte concentrations and fluorescent intensities. Both calibration plots exhibited well-defined linear relationships between the concentration and fluorescent intensity. The sensitivity for BLCA-4 detection was $2 \mathrm{U} / \mathrm{ml}$ and for NMP52 detection was $1 \mu \mathrm{g} / \mathrm{ml}$.

\section{Accuracy and precision assay}

Three clinical samples with low, median and high concentrations of BLCA-4 and NMP52 were analyzed to calculate the accuracy and precision of this assay. As shown in Table 1, for BLCA-4 detection, the inter-assay CVs ranged from $6.9 \%$ to $7.4 \%$, and intra-assay CVs ranged from $5.1 \%$ to $6.7 \%$. For NMP52 detection, the inter-assay CVs ranged from $6.6 \%$ to $8.5 \%$, and intra-assay CVs ranged from $5.8 \%$ to $6.3 \%$. Therefore, the low CVs (less than 10\%) demonstrating this assay as a good performance.

\section{Recovery assay}

In the present study, 5 clinical urine samples with known concentrations of BLCA-4 and NMP52 that spiked with different amounts of BLCA-4 and NMP52 standards were performed. In clinical samples, the initial concentrations of BLCA-4 were $10.4 \mathrm{U} / \mathrm{mL}, 24.6$ $\mathrm{U} / \mathrm{mL}, 51.5 \mathrm{U} / \mathrm{mL}, 102.7 \mathrm{U} / \mathrm{mL}, 263.8 \mathrm{U} / \mathrm{mL}$, and for NMP52, the initial concentrations were $5.6 \mu \mathrm{g} / \mathrm{ml}, 15.4 \mu \mathrm{g} / \mathrm{ml}, 42.1 \mu \mathrm{g} / \mathrm{ml}, 88.9 \mu \mathrm{g} /$ $\mathrm{ml}, 135.7 \mu \mathrm{g} / \mathrm{ml}$. For BLCA-4, the average recoveries ranged from $95.1 \%$ to $108.7 \%$, and for NMP52, the average recoveries ranged from $94.6 \%$ to $105.2 \%$ (Table 2). These data indicated that the present dual-labeled TRFIA was independent of urine interferent.

\section{Specificity assay}

To detect the specificity of this assay, some potential various concentrations of interferents were analyzed. No cross-reactivity was determined among these molecules (Table 3 ). The data suggested that the present dual-labeled TRFIA has high affinity and specificity for BLCA-4 and NMP52.

\section{Comparison with commercialized kits}

To carry out the parallel tests, 80 clinical samples were analyzed using the present dual-label TRFIA and Roche ELISA methods. The results observed that high correlations between the two methods were obtained. For BLCA-4, the linear equation was shown as $y=0.971 \mathrm{x}-7.845$, and the correlation coefficient was $0.99(\mathrm{P}<0.001)$ (Figure 3). For NMP52, the linear equation was shown as $\mathrm{y}=0.863 \mathrm{x}-0.732$, and the correlation coefficient was $0.99(\mathrm{P}<0.001)$. These observations indicated that the present dual-label TRFIA was a reliable method for the simultaneous detection of BLCA-4 and NMP52 in clinical samples.

\section{Clinical urine sample analysis}

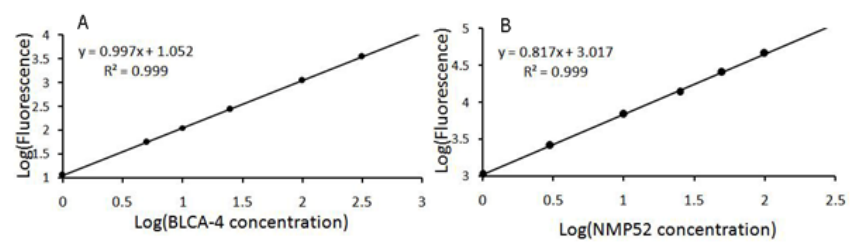

Figure 2: Calibration plots for BLCA-4 (A) and MNP52 (B) detection.

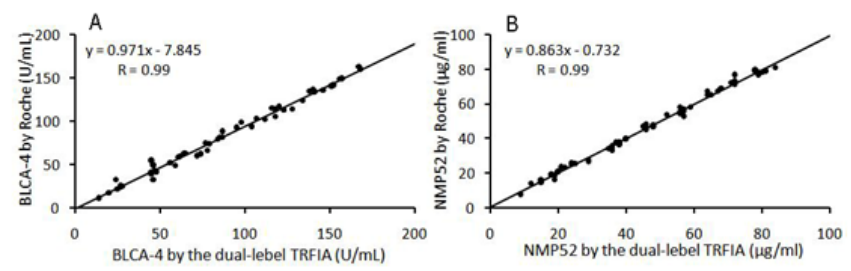

Figure 3: Correlation of BLCA-4 (A) and MNP52 (B) results between TRFIA and ELISA.

Significant differences of BLCA-4 and NMP52 urine levels between benign and malignant cases for the whole group were found (Table 4). In benign patients, the median BLCA-4 level was $37.2 \mathrm{U} / \mathrm{mL}$, and was much less than that of malignant patients $(100.1 \mathrm{U} / \mathrm{mL},<0.01)$. The median NMP52 levels in benign patients and malignant patients were $9.2 \mu \mathrm{g} / \mathrm{ml}$ and $43.7 \mu \mathrm{g} / \mathrm{ml}$ respectively, which were significant differences within the two groups. There were significant differences for BLCA-4 and NMP52 between low grade (Ta-T2) and high grade $(\mathrm{T} 3,<0.01)$ of tumors. In stage T3 tumors, levels of BLCA-4 and NMP52 were lower than those in stage $\mathrm{T} 4$ tumors in urine. However, there was no difference exist among low grade (Ta-T2) $(>0.05)$.

\section{Discussion}

Bladder cancer is the second most common genitourinary malignant disease in the USA $[21,22]$. The high risk of recurrence makes effective and sensitive detection means to be very importance for bladder cancer patients $[9,23]$. Conventional methods for bladder cancer detection such as morphological examination of cytology samples or cystoscopic biopsies, is time-consuming and poor discrimination. Here, we developed a dual-label TRFIA for bladder cancer determination in a single run using BLCA-4 and NMP52 as biomarkers.

TRFIA represent an ultrasensitive technique using lanthanide elements and their chelates as the tracer with unique fluorescence properties. Compared with other immunoassay methods, such as, radioimmunoassay (RIA), enzyme immunoassay (EIA) and chemiluminescence immunoassay (CLIA), TRFIA is safe, highly sensitive, and has a wide dynamic range (19). Furthermore, the labeling procedure is very easy and produces labelled compounds with high specific activity and good stability with minimal influence on immunoreactivity and other bioactivities. Therefore, TRFIA has better performance in urine sample analysis than traditional fluoro immunoassays. In addition, TRFIA has been widely used in various fields, such as microbiology molecular biology, drug analysis and clinical research [24].

At present, many of the bladder tumor markers from serum and urine were reported and some of them had been developed for disease diagnosis. For example, Shahrokh et al. combined results from 10 centers and 2542 patients to develop internally validated nomograms to predict bladder cancer recurrence [25]. In this model, NMP22 had independent predictive power to predict bladder cancer recurrence and progression. BTA-stat tests detect complement factor $\mathrm{H}$ and a complement-factor $\mathrm{H}$-related protein in urine. Cheng et al. had identified the binding sites of 2 monoclonal antibodies that are used in the BTA-stat tests [26]. However, the emerging evidences found that there were many disadvantages in some bladder tumor markers. Investigations revealed that NMP22 has no predictive value for bladder cancer recurrences, the specificity of NMP22 ELISA was low (between $60 \%$ and $80 \%$ ) and a high false-positive rate among patients with inflammatory conditions, renal or bladder calculi, foreign bodies, bowel interposition and protein urea $[25,27-$ 
Citation: Liu X, Li Y, Wang Y, Sun W, Li L, et al. (2017) Development of a Dual-Label Time-Resolved Fluorescence Immunoassay (TRFIA) for Screening of Bladder Cancer based on Simultaneous Detection of BLCA-4 and NMP52 in Urine. J Bioprocess Biotech 7: 303. doi:10.4172/21559821.1000303

Page 4 of 5

\begin{tabular}{|c|c|c|c|c|}
\hline & \multicolumn{4}{|c|}{ BLCA-4 } \\
\hline & Concentration & Mean (U/ml) & Standard deviation (SD) & Variable coefficient (CV) \\
\hline \multirow{3}{*}{ Inter-assay } & low & 14.9 & 1.1 & $7.4 \%$ \\
\hline & median & 142.8 & 9.9 & $6.9 \%$ \\
\hline & high & 286.5 & 21.0 & $7.3 \%$ \\
\hline \multirow{5}{*}{ Intra-assay } & low & 13.8 & 0.9 & $6.5 \%$ \\
\hline & median & 150.1 & 10.1 & $6.7 \%$ \\
\hline & high & 290.6 & 14.8 & $5.1 \%$ \\
\hline & \multicolumn{4}{|c|}{ NMP52 } \\
\hline & Concentration & $\begin{array}{l}\text { Mean } \\
(\mu \mathrm{g} / \mathrm{ml})\end{array}$ & Standard deviation (SD) & Variable coefficient (CV) \\
\hline \multirow{3}{*}{ Inter-assay } & low & 9.4 & 0.8 & $8.5 \%$ \\
\hline & median & 80.8 & 6.1 & $7.6 \%$ \\
\hline & high & 145.5 & 9.6 & $6.6 \%$ \\
\hline \multirow{3}{*}{ Intra-assay } & low & 11.1 & 0.7 & $6.3 \%$ \\
\hline & median & 77.9 & 5.1 & $6.5 \%$ \\
\hline & high & 138.6 & 8.1 & $5.8 \%$ \\
\hline
\end{tabular}

Table 1: Accuracy and precision of the dual-label TRFIA $(n=10)$.

\begin{tabular}{|c|c|c|c|c|c|c|}
\hline \multirow[b]{2}{*}{ Samples } & \multicolumn{2}{|c|}{ BLCA-4 } & \multirow[b]{2}{*}{ Recovery $(100 \%)$} & \multicolumn{2}{|c|}{ NMP52 } & \multirow[b]{2}{*}{ Recovery $(100 \%)$} \\
\hline & $\begin{array}{l}\text { Original concentration } \\
\qquad(\mathrm{U} / \mathrm{mL})\end{array}$ & $\begin{array}{l}\text { Measured Value (U/ } \\
\mathrm{mL})\end{array}$ & & $\begin{array}{c}\text { Original } \\
\text { concentration }(\mu \mathrm{g} / \\
\mathrm{mL})\end{array}$ & $\begin{array}{l}\text { Measured Value } \\
(\mu \mathrm{g} / \mathrm{mL})\end{array}$ & \\
\hline 1 & 10.4 & 11.3 & 108.7 & 5.6 & 5.3 & 94.6 \\
\hline 2 & 24.6 & 24.2 & 98.4 & 15.4 & 16.2 & 105.2 \\
\hline 3 & 51.5 & 53.8 & 104.5 & 42.1 & 43.9 & 104.3 \\
\hline 4 & 102.7 & 105.2 & 102.4 & 88.9 & 86.1 & 96.6 \\
\hline 5 & 263.8 & 250.9 & 95.1 & 135.7 & 136.2 & 103.7 \\
\hline Mean \pm SD & & & $101.8 \pm 4.2$ & & & $100.9 \pm 3.9$ \\
\hline
\end{tabular}

Table 2: Recoveries of the dual-label TRFIA $(n=10)$.

\begin{tabular}{|c|c|}
\hline Compound & \% Cross-reactivity \\
\hline Thyroid Hormones & 0.3 \\
\hline Luteinizing Hormone & 0.1 \\
\hline Testosterone & 0.05 \\
\hline Follicle Stimulating Hormone & 0.1 \\
\hline Cortisol & 0.01 \\
\hline Estradiol & 0.02 \\
\hline
\end{tabular}

Table 3: Cross-reactivities of the dual-label time-resolved fluorescence immunoassay $(n=10)$.

\begin{tabular}{|c|c|c|c|c|c|}
\hline \multirow{2}{*}{} & \multirow{2}{*}{ Number } & \multicolumn{2}{|c|}{ BLCA-4 $\mathbf{( U / m L})$} & \multicolumn{2}{c|}{ NMP52 $(\boldsymbol{\mu g} / \mathbf{m l})$} \\
\cline { 3 - 6 } & & Median & Range & Median & Range \\
\hline Normal control & 40 & 8.5 & $3.1-13.8$ & 1.5 & $1.1-1.8$ \\
\hline cystitis & 54 & 32.2 & $20.5-43.8$ & 4.0 & $3.3-4.6$ \\
\hline Benign & 126 & 37.2 & $24.8-49.6$ & 9.2 & $6.2-12.2$ \\
\hline Malignant & 180 & 100.1 & $29.7-170.5$ & 43.7 & $12.6-74.8$ \\
\hline Ta & 25 & 40.5 & $26.3-54.6$ & 12.1 & $8.4-15.8$ \\
\hline T1 & 21 & 44.9 & $30.0-59.7$ & 14.4 & $9.8-18.9$ \\
\hline T2 & 98 & 51.9 & $35.9-67.8$ & 18.6 & $13.7-23.4$ \\
\hline T3 & 80 & 123.6 & $77.6-169.5$ & 49.8 & $17.8-81.8$ \\
\hline T4 & 82 & 107.3 & $60.2-154.3$ & 26.1 & $11.5-40.6$ \\
\hline
\end{tabular}

Table 4: Urinary BLCA-4 and NMP52 levels in clinical samples.

29]. Also, low sensitivity been found in BTA-stat tests, ranges from $8 \%$ to $89 \%$, especially, for low-grade tumors detecting [30]. The specificity of BTAstat tests is low among patients with benign urologic conditions, especially those that cause hematuria and small recurrent tumors [26,31].

In this study, we developed a TRFIA for bladder cancer detection using novel urinary biomarkers BLCA-4 and NMP52. Well-defined linear relationships between the analyte concentrations and fluorescent intensities with broad detection ranges were revealed in this assay. High correlations with commercial ELISA kits made this assay as a good alternative to detect bladder cancer. Moreover, the urinary level of BLCA-4 and NMP52 form the clinical sample analysis was correlated with the stages of tumors.

In summary, the present dual-label TRFIA, allowing the simultaneous detection of BLCA-4 and NMP52, has high sensitivity, specificity, and accuracy in clinical sample analysis. It is helpful for the early diagnosis and prognosis monitoring of bladder cancer patients. Furthermore, it also could lower the economic burden to the bladder cancer patients. Therefore, it has good prospects of application.

\section{Acknowledgments}

Thanks for the Zhujiang Hospital and Jinan Hospital for supplying urine samples from patients with pathologically confirmed bladder cancer, cystitis patients and normal controls, and Guangdong province science and technology plan projects (20150119).

\section{References}

1. Donald K, Adam F (2009) Bladder cancer. Lancet 374: 239-249.

2. Olaf PJ, Vrooman JAW (2008) Urinary Markers in Bladder Cancer. European urology 53: 909-916.

3. Rebecca JM, Zou ZH, Ahmedin J, DVM (2014) Cancer Statistics 2014. CA Cancer J Clin 64: 9-29.

4. Ziya TC, Murugesan M, Ferean A, Christer B, Lianng C, et al. (2005) Bladder cancer: epidemiology, staging and grading, anddiagnosis. Urology 66: 4-34. 
Citation: Liu X, Li Y, Wang Y, Sun W, Li L, et al. (2017) Development of a Dual-Label Time-Resolved Fluorescence Immunoassay (TRFIA) for Screening of Bladder Cancer based on Simultaneous Detection of BLCA-4 and NMP52 in Urine. J Bioprocess Biotech 7: 303. doi:10.4172/21559821.1000303

5. Farrow GM (1990) Urine cytology in the detection of bladder cancer: a critical approach. Journal of Occupational Medicine 32: 817-821.

6. Messing D (2009) Commentary: the role of cytologic analysis of voided urine in the work-up of asymptomatic microhematuria. BMC Urology 9: 13

7. Tetu B (2009) Diagnosis of urothelial carcinoma from urine. Mod Pathol 22: S53-S59.

8. Steve CJ, Rosser, Virginia U (2013) Bladder Cancer Detection and Monitoring Assessment of Urine- and Blood-Based Marker Tests. Mol Diagn Ther 17: 71-84.

9. Vinata L, Marie S (2006) Urinary bladder tumor markers. Urologic Oncology: Seminars and Original Investigations 24: 528-537.

10. Attallah M, Sanaa A, El-Waseef, Omran MM, Saadh MJ, et al. (2015) Diagnostic Performance of Nuclear Matrix Protein (NMP-52) in Bladder Cancer. Global Journal of Biotechnology and Biochemistry 10: 32-38.

11. Badrinath RK, Rajiv D, Roger SD, Michael JB, Walter MS, et al. (2000) Detection of Bladder Cancer Using a Novel Nuclear Matrix Protein, BLCA-4. Clin Cancer Res 6: 2618-2625.

12. Feng CC, Guan M, Jian HW, Wen H, Ding Q, et al. (2011) Urinary BLCA-4 is Highly Specific for Detection of Bladder Cancer in Chinese Han Population and Is Related to Tumor Invasiveness. Folia Biologica (Praha) 57: 242-247.

13. Xie MJ, Hang JF, Dong ZN, Xiao DY, Xu P, et al. (2015) Evaluation of the Analytical and Clinical Performances of Time-resolved Fluoroimmunoassay for Detecting Carcinoma Antigen 50. J Immunoassay Immunochem 3: 265-283.

14. Julie MMI, TS Van le, Robert HG (2005) Mechanistic Analysis of the Role of BLCA-4 in Bladder Cancer Pathobiology. Cancer Res 65: 7145-7150.

15. Van TS, Badrinath RK, Timothy B, Robert HG (2004) Functional Characterization of the Bladder Cancer Marker, BLCA-4. Clin Cancer Res 10: 1384-1391.

16. Rebecca DN, Ahmedin J (2012) Cancer Statistics, 2012. CA Cancer J Clin 62 10-29.

17. Abdelfattah AH, Hisham I, Elsayked A, Ibrahim E (2005) An office-based immunodiagnostic assay for detecting urinary nuclear matrix protein 52 in patients with bladder cancer. BJU Int 96: 334-339.

18. Attallah AM, Abdallah SO, El-Waseef AM, Omran MM, Abdelrazek MA, et al. (2015) Combined use of epithelial membrane antigen and nuclear matrix protein 52 as sensitive biomarkers for detection of bladder cancer. Int J Biol Markers 4: $32-41$
19. Liu TC, Ren ZQ, Hou JY, Lin GF, Wu YS (2014) Development of an improved time-resolved fluoroimmunoassay for simultaneous quantification of C-peptide and insulin in human serum. Clin Biochem 47: 439-444.

20. Lin GF, Zou LP, Hou JY, Wu YS (2013) Development of a dual-label timeresolved fluoroimmunoassay for the detection of a-fetoprotein and hepatitis $B$ virus surface antigen. Luminescence 28: 401-406

21. Saniay JB, Jennifer AB, Steven GR, Peter CW, Michael LB, et al. (1999) Comparison of screening methods in the detection of bladder cancer. J Urol 161: 388-394.

22. Thu-Suong VL, Timothy B, Marko B, Douglas MP, Robert HG (2005) Highlyspecificusing-based markerofbladder cancer. Urology 66: 1256-1260.

23. Willem BL, Gerhard J, Malmstrom PU, Michael S, Cora S (2002) Guidelines on Bladder Cancer. European Urology 41: 105-112.

24. Patra D, Barakat C (2012) Time-resolved fluorescence study during denaturation and renaturation of curcumin-myoglobin complex. Int J Biol Macromol 50: 885-890.

25. Shahrokh FS, Yair L, Marta SC, Craig Z, Gerson L, et al. (2006) Variability in the Performance of Nuclear Matrix Protein 22 for the Detection of Bladder Cancer. J Urol 176: 919-926.

26. Cheng ZZ, Maria P, Sandra M, Jens H, Peter FZ, et al. (2005) Complement Factor $\mathrm{H}$ as a Marker for Detection of Bladder Cancer. Clin Chem 51: 856-863.

27. Christine ML, Andrea H, Eduard EV, Michael M, Armin P (2000) Comparison of themonoclonal UBC-ELISA testand the NMP22 ELISA testfor the detectionof urothelialcellcarcinoma of the bladder. Urology 55: 223-226.

28. Lee Ponsky SS, Lakshmi P, Sumita K, David N, Ashok A, et al. (2001) Screening and monitoring for bladder cancer: Refining the use of NMP22. J Urol 166: 75-78.

29. Sanchez MU, Silva M, Romanni R, Navajo JA (2001) Comparative predictive values of urinary, urinary bladder cancer antigen, CYFRA 21-1 and NMP22 for evaluating symptomatic patients at risk for bladder cancer. J Urol 165: 1462-1467.

30. Hans BH (2002) Four Bladder tumor markers have a disappointingly low sensitivity for small size and low grade recurrence. J Urol 167: 80-83.

31. Vinata BL, Grossman HB, William MM, Stefan HH, George PH, et al. (2005) Bladder tumor markers beyond cytology: International consensus panel on bladder tumor markers. Urology 66: 35-63. 